\title{
Quantum spin chains with various defects
}

\author{
Cosima Schuster* and Ulrich Eckern \\ Institut für Physik, Universität Augsburg, 86135 Augsburg, Germany \\ * Corresponding author: cosima.schuster@physik.uni-augsburg.de
}

\begin{abstract}
Using the density matrix renormalization group (DMRG) method, we study the quantum coherence in one-dimensional disordered spin chains and Fermi systems. We consider in detail spinless fermions on a ring, and compare the influence of several kinds of impurities in a gapless and a dimerized, gapped system. In the translation-invariant system a so-called site-impurity, which can be realized by a local potential or a modification of one link, increases for repulsive interaction, and decreases for attractive interaction, upon renormalization. The weakening of two neighbouring bonds, which is a realization of a socalled bond-impurity, on the other hand, is healed for repulsive interaction, but enhanced for intermediate attractive interactions. This leads to a strong suppression of the quantum coherence measured by the phase sensitivity, but not to localization. Adding a local distortion to a dimerized system, we find that even the presence of a single site-impurity increases the metallic region found in the dimerized model. For a strong dimerization and a high barrier, an additional sharp maximum, is seen in the phase sensitivity as a function of interaction, already for systems with about 100 sites. A bond-impurity in the dimerized system also opens a small metallic window in the otherwise isolating regime.
\end{abstract}

Keywords: quantum spin systems, disordered wires

PACS: $75.10 . \mathrm{Jm}, 75.40 . \mathrm{Ng}$

\section{Introduction}

Recent experiments have led to a renewed theoretical interest in disordered spin-Peierls systems. For example, the doped one-dimensional Heisenberg system $\mathrm{Cu}_{1-x} \mathrm{Zn}_{x} \mathrm{GeO}_{3}$ [1] shows two subsequent magnetic transitions, the spin-Peierls transition at $T=14$ $\mathrm{K}$ followed by a transition to an antiferromagnetic ground-state at $T_{\mathrm{N}}=5 \mathrm{~K}$, while in $\mathrm{Cu}_{1-x} \mathrm{Mg}_{x} \mathrm{GeO}_{3}$, a reentrant spin-Peierls phase for $x>x_{c}$ [2] is observed. In fact, the general question of the effect of various types of impurities in pure [3, 4, 旬, 6, 6, 8] or gapped spin systems 19, 10, 11 has been studied intensively during the last years. Nevertheless, the interplay between interaction, disorder, and periodic distortions is still a challenging problem. The model of spinless fermions, which is considered here in detail, is equivalent to the anisotropic Heisenberg model. It describes certain aspects of magnetic and electronic systems, and the phase diagram of this "simplest" interacting fermion model is surprisingly rich. On the basis of this model, the aim of this work is thus to achieve a better understanding of the ground-state properties of disordered spin- and interacting Fermi-systems, especially clarifying the role of the interaction, which may enhance or decrease the localization due to the random and periodic perturbations. In particular, we will introduce and compare the effects of 
various kinds of impurities.

In this context, Eggert and Affleck [3] pointed out that two "classes" of impurities exist, which differ in their effect on the local symmetry of the system. So-called "site-impurities" violate the site parity by affecting one site or bond, whereas "bondimpurities", which modify two neighbouring bonds, violate the bond symmetry but respect the site parity. Bearing this in mind, we use the name site- or bond-impurity. Note that this naming is different from the one used in [4] and [5], where an impurity on a single site is called site-impurity, and an impurity on a bond is called bondimpurity. Another type, "transparent" impurities, which we do not consider here, can be constructed using the Bethe-Ansatz [6]: Those defects are similar to bondimpurities, but include also a coupling between the next-nearest neighbours around the impurity. Transparent impurities have also been studied extensively with analytic and numerical techniques during the last years, see for example [7] and [8]. As a result of Eggert and Affleck, a site-impurity is relevant in the sense that it can break up a closed ring, whereas a bond-impurity is irrelevant, i. e. the defect is healed at low energies.

Gapped spin systems containing irrelevant impurities, i. e. impurities which do not close the gap in the system, are supposed to be equivalent to free Dirac-fermions with random mass, a model which has been widely investigated in one dimension [12, 13] in the context of doped spin-Peierls or spin-ladder systems and two dimensions [14] in the context of the Quantum Hall Effect. However, the transformation to Dirac fermions is valid only for special points in the parameter space, which correspond to the XY model (free fermions) for the dimerized system, or the isotropic XXXHeisenberg antiferromagnet for the ladder model.

The numerical density matrix renormalization group 15 is a quasi-exact numerical method to determine the ground state properties, in particular the ground-state energy, of long one-dimensional (non-integrable) systems with reasonable accuracy. The bosonization technique [16] can be used with advantage to interpret the numerical data.

In the following, we introduce the models and the impurity types studied by us. Using the bosonization technique and the Luttinger description, we then identify the leading non-linear operators, and classify the impurities according to them. The results for single defects are presented in Sec. 3. Concerning the dimerized system, we concentrate in our discussion on strong site- and bond-impurities. The results are discussed in Sec. 囷, followed by a summary in Sec. 5 .

\section{Magnetic chains and spinless fermions}

We begin by presenting the generic spin model, and then describe the equivalent fermionic model. Then we briefly introduce the Luttinger description, which is useful for discussing the relevant operators which lead to insulating behaviour. 


\subsection{The Heisenberg spin chain}

As a starting point for the study of a general disordered spin-Peierls system in one dimension, we consider an anisotropic Heisenberg chain, given by the XXZ model, with a dimerized interaction:

$$
H_{\text {spin }}=-\sum_{i=1}^{N} J_{i}(u)\left(\sigma_{i}^{x} \sigma_{i+1}^{x}+\sigma_{i}^{y} \sigma_{i+1}^{y}+\Delta \sigma_{i}^{z} \sigma_{i+1}^{z}\right)+N \frac{K}{2} u^{2},
$$

where the dimerization in the Peierls state, $u$, enters the coupling constant according to $J_{i}(u)=J\left[1+(-)^{i} u\right]$. For the clean XXZ model, i. e. for $u=0$, and for zero total magnetisation, $M=\sum_{i}\left\langle\sigma_{i}^{z}\right\rangle=0$, one finds three phases: a ferromagnetic phase for $\Delta \geq 1$, separated by a first-order transition from a gapless phase for $-1 \leq \Delta<1$, whose low lying excitations are given by those of a Luttinger liquid; and an antiferromagnetic phase for $\Delta<-1$. The transition from the Luttinger to the antiferromagnetic phase is of Berezinskii-Kosterlitz-Thouless type. Adding the dimerization, $u$, the system becomes localized by forming spin-singlets on neighbouring sites for $\Delta<0$, i.e. for antiferromagnetic coupling. An excitation gap opens due to the usual Peierls mechanism [17]. Leaving aside the question whether a finite $u$ can be stabilized, we note that the dimerization is already relevant for $\Delta<\sqrt{2} / 2$ [18, 19], and the ground-state wave function is localized. The interaction-dimerization phase diagram was determined in [20].

Impurities may be realized in three different ways. First, local magnetic fields may be present, resulting for example from magnetic impurities near the chain, which couple directly to the $\sigma^{z}$-component at a specific site. We call this kind of impurity a site-impurity. Second, the coupling between the spins may be modified locally by a factor $(1-\delta)$, for example by substituting $\mathrm{Sr}$ with La or Ge with $\mathrm{Si}$ in a spin chain compound like $\mathrm{La}_{x} \mathrm{Sr}_{2-x} \mathrm{CuO}_{3}$ or $\mathrm{CuGe}_{1-x} \mathrm{Si}_{x} \mathrm{O}_{3}$. As only one link is concerned this is similar to a local field; nevertheless we call it link-impurity in the following. The third possibility is to modify both bonds to the left and to the right of the impurity site by an equal amount, $(1-b)$ : This situation, which is called bond-impurity, can be realized by doping magnetic impurities with spin $1 / 2$ into a chain, e. g. substituting $\mathrm{V}$ by $\mathrm{Nb}$. (Nevertheless, in some cases it is found that the free Nb-electron - with the spin - moves to the Vanadium site, i. e. a $\mathrm{Nb}^{5+}$ - and a $\mathrm{V}^{3+}$-site is established instead of $\mathrm{Nb}^{4+}$ and $\mathrm{V}^{4+}$.) Completing the Hamiltonian (11) in this way, we can write

$$
H_{\text {spin }}=-\sum_{i=1}^{N} J_{i}(u, \delta, b)\left(\sigma_{i}^{x} \sigma_{i+1}^{x}+\sigma_{i}^{y} \sigma_{i+1}^{y}+\Delta \sigma_{i}^{z} \sigma_{i+1}^{z}\right)-\sum_{i=1}^{N} h_{i} \sigma_{i}^{z},
$$

with $J_{i}(u, \delta, b)=J_{i}(u)\left[1-\delta_{i, m} \delta-\left(\delta_{i, m}+\delta_{i, m+1}\right) b\right]$, where $m$ is a fixed site.

\subsection{The fermionic model}

The corresponding fermionic model is obtained via the Jordan-Wigner transformation. In the result we change the notation, $J \rightarrow t, J \Delta \rightarrow-V / 2$, and $\epsilon_{m} \rightarrow-2 h_{m}$, and neglect constant energy shifts, like $\sum_{i} h_{i}$ and $\Delta\left(2 N_{f}-N / 2\right)$, where $N_{f}$ is the number 
of fermions:

$$
\begin{aligned}
H_{\text {fermion }}= & -\sum_{i} t_{i}\left(c_{i}^{+} c_{i+1}+c_{i+1}^{+} c_{i}\right)+\sum_{i} V_{i} n_{i} n_{i+1}+\sum_{i} \epsilon_{i} n_{i} \\
& -\frac{V}{2}\left[\delta\left(n_{m}+n_{m+1}\right)+b\left(n_{m-1}+2 n_{m}+n_{m+1}\right)\right],
\end{aligned}
$$

where $t_{i}(u)=t\left[1+(-1)^{i} u_{t}-\delta_{i, m} \delta_{t}-\left(\delta_{i, m}+\delta_{i, m+1}\right) b_{t}\right]$, and $V_{i}(u)=V\left[1+(-1)^{i} u_{V}-\right.$ $\left.\delta_{i, m} \delta_{V}-\left(\delta_{i, m}+\delta_{i, m+1}\right) b_{V}\right]$. Furthermore, we have assumed that the coupling to (static) phonons and to the impurities can be varied independently in both the hopping and the interaction, by introducing six parameter $\left(u_{t}, u_{V}, \delta_{t}, \delta_{V}, b_{t}, b_{V}\right)$ instead of three $(u, \delta, b)$. The particle density is $n_{0}=N_{f} / N$, and we restrict ourselves to half filling; we set $t=1$ in some of the formulas below. As shown in [21], $u_{t}$ is the main contribution arising from the dimerization, whereas $u_{V}$ modifies the results only quantitatively but not qualitatively; thus we assume $u_{V}=0$. For simplicity, we likewise neglect (1) to avoid the mixing - in the fermionic picture - of bond- and site-impurity. In the case of the link-impurity, it was shown by Meden et al. [5] that the weakening of one bond in the spin model is equivalent to an impurity given by $\delta_{t}$ and $\delta_{V}$, i. e. that the contribution (4) is of lesser importance. We assume that the same holds for the bond-impurity. The in this way simplified Hamiltonian is then given by

$$
\begin{aligned}
H_{\text {fermion }}= & -\sum_{i} t\left[1+(-1)^{i} u_{t}-\delta_{i, m} \delta_{t}-\left(\delta_{i, m}+\delta_{i, m+1}\right) b_{t}\right]\left(c_{i}^{+} c_{i+1}+c_{i+1}^{+} c_{i}\right) \\
& +\sum_{i} V\left[1-\delta_{i, m} \delta_{V}-\left(\delta_{i, m}+\delta_{i, m+1}\right) b_{V}\right]+\sum_{i} \epsilon_{i} n_{i} .
\end{aligned}
$$

Thus the link-impurity can occur as a pure hopping-impurity, $\delta_{t} \neq 0$ and $\delta_{V}=0$, or a generic link-impurity, $\delta_{t} \neq 0$ and $\delta_{V} \neq 0$. Setting $\delta_{t}=0$, we can also realize an interaction-impurity by varying $\delta_{V}$. In the following we consider the Hamiltonian given by Eq. (5) only.

\subsection{Bosonization and Luttinger description}

For the interpretation of the numerical data, we use the fact that in the gapless phase the system can be described by a Luttinger liquid, and that the distortions can be considered as a perturbation. In the bosonized form [16, the Hamiltonian can be written for the "clean" model as follows:

$$
H_{\text {boson }}=\int \frac{\mathrm{d} x}{2 \pi}\left\{\frac{v}{g}\left[\partial_{x} \varphi(x, t)\right]^{2}+v g\left[\pi \Pi_{\varphi}(x, t)\right]^{2}\right\},
$$

where $\Pi_{\varphi}$ is the momentum density conjugate to $\varphi(x, t)$, i. e. it corresponds to $\Pi_{\varphi}=$ $\partial_{t} \varphi /(v g)$ in the (Euclidean) path integral formulation of the theory. It is also related to the conjugate phase variable $\theta$ through $\Pi_{\varphi}=\partial_{x} \theta(x) / \pi$. The velocity $v$ of the bosonic excitations is given by $v=[\pi t \sin (2 \eta)] /(\pi-2 \eta)$, and the interaction constant is $g=\pi / 4 \eta$, where $\eta$ parameterizes the interaction according to $V=-2 t \cos (2 \eta)$. The density (operator) is given by

$$
n(x)=n_{0}+\frac{\partial \varphi}{\pi \partial x}+\frac{k_{F}}{\pi} \cos \left[2 k_{F} x+2 \varphi(x)\right] .
$$


We chose the above standard representation because then the order of the scattering process is directly seen in the non-linear terms. Non-linear - global and local - contributions arise from the dimerization and the interaction as well as from the impurities.

- Dimerization causes a $2 k_{F}$-scattering process of the fermions,

$$
H_{u} \propto 2 u \int \mathrm{d} x \sin [2 \varphi(x)] .
$$

- While the fermion-fermion scattering with $q \approx 0$ and $q \approx 2 k_{F}$ is absorbed in the Luttinger-parameter $g$, the $4 k_{F}$-scattering leads to

$$
H_{V} \propto V \int \mathrm{d} x \cos \left[4 \varphi(x)+\left(4 k_{F} x-G\right) x\right],
$$

where $G$ is the reciprocal lattice vector. This term causes the transition to the CDW-state for $V=2(g=1 / 2)$.

- Backscattering arises from a local potential (say at $x=0$ ). Since the potential couples directly to the density, Eq. (7), we find in lowest order in the impurity strength

$$
H_{\epsilon}=\epsilon_{0} n_{0} \propto \epsilon_{0} n_{2 k_{F}}(x=0) \propto \epsilon_{0} \cos [2 \varphi(0)] .
$$

A strong potential in an infinitely long chain is equivalent to a weak link [22]: The diagonalization of the above Hamiltonian, $H_{\text {fermion }}+H_{\epsilon}$, leads, in the case of a strong potential between two semi-infinite chains ( $R$ : right chain, $L$ : left chain), to the contribution

$$
H_{\epsilon} \propto \frac{c_{R}^{+}(0) c_{L}(0)}{\epsilon_{0}} \propto \frac{1}{\epsilon_{0}} \cos [2 \theta(0)] .
$$

- A change in the hopping is also considered in the weak, $\delta_{t} \rightarrow 0$, and strong, $\delta_{t} \rightarrow 1$, limit. Following the analysis for the (weak) periodic [21] or random potential [23], we would assume that the transition from a site-centered potential to a bond-centered potential causes only a phase shift of $\pi / 2$ in the non-linear term, i. e. a shift from a cosine to a sine. The weak link thus should correspond to a high barrier, see again [22], i. e.

$$
\begin{aligned}
& H_{t} \propto \delta_{t} \sin [2 \varphi(0)], \quad \delta_{t} \rightarrow 0 \\
& H_{t} \propto\left(1-\delta_{t}\right) \cos [2 \theta(0)], \quad \delta_{t} \rightarrow 1
\end{aligned}
$$

should be appropriate descriptions. However, if we consider a modified link with $H_{t} \propto\left(1-\delta_{t}\right)\left(c_{1}^{+} c_{0}+\right.$ h. c. $)$, we find instead

$$
\begin{aligned}
& H_{t} \propto \delta_{t} \sin [\varphi(1) \sin [\varphi(0)] \\
& H_{t} \propto\left(1-\delta_{t}\right) \cos [\theta(1)-\theta(0)] .
\end{aligned}
$$


- The local modification of the interaction, i. e. the interaction-impurity, leads to a $2 k_{F}$-contribution too, in addition to the $4 k_{F}$-term already present in Eq. (9):

$$
H_{\delta_{V}} \propto H_{t}
$$

- The modification of two links, in the limit of $b=b_{t}=b_{V} \rightarrow 0$, does not cause $2 k_{F}$-scattering at half filling. Thus, no non-linear contribution to the Hamiltonian is found. In the limit $b \rightarrow 1$, however, we find the following expression:

$$
\begin{aligned}
H_{b}= & (1-b)\left(c_{1}^{+} c_{0}+c_{0}^{+} c_{-1}+\text { h.c }\right) \\
\propto & (1-b) \sin \{[\theta(1)+\theta(-1)] / 2-\theta(0)\} \sin \{[\theta(1)-\theta(-1)] / 2\} \\
& \quad \times \cos [\varphi(0)] \sin \{[\varphi(1)+\varphi(-1)] / 2\} \\
\propto & (1-b) \sin (\Phi / 2) \sin [\Theta(1)] \cos [\varphi(0)] \sin [\phi(1)] .
\end{aligned}
$$

In the last step we use $\theta(x)=\theta_{0}+\Phi x / L+\tilde{\theta}(x)$, see [16], where $\theta_{0}$ represents the zero-mode contribution, $\Phi x / L$ the boundary condition - see also the following section -, and $\tilde{\theta}(x)$ the excitations. In addition, the abbreviations $\varphi(x)+\varphi(-x)=$ $2 \phi(x)$, and $\theta(x)-\theta(0)=\Theta(x)$ are used.

\subsection{The phase sensitivity}

We will use the phase sensitivity, i. e. the reaction of the system to a change in the boundary condition, to determine the localized-delocalized transition numerically for systems with finite size. We model the different boundary condition via a magnetic flux, penetrating the ring of the spinless fermions. The effect of the magnetic flux results in an additional phase of the hopping terms, $t_{j} \rightarrow\left|t_{j}\right| \mathrm{e}^{i \Theta_{j}}$, with $-\pi<\Theta_{j}<\pi$. The energy levels depend only on the total flux, $\Phi=\arg \left(\Pi_{j=1}^{N} t_{j}\right)$. In particular, we determine below the energy difference between periodic $\left(c_{N}=c_{0}, \Phi=0\right)$ and anti-periodic $\left(c_{N}=-c_{0}, \Phi=\pi\right)$ boundary conditions, $\Delta E=(-)^{N_{f}}(E(0)-E(\pi))$. Here the factor $(-1)^{N_{f}}$ cancels the odd-even effects resulting from the change in the ground-state for odd versus even particle numbers. We recall that, for a clean system, the ground-state energy and the finite-size corrections can be obtained from the Bethe Ansatz [24]. At half filling (and for odd particle number), the result, in the Luttinger regime, is given by 25

$$
E_{N}(\Phi)-N \varepsilon_{\infty}=-\frac{\pi v}{6 N}\left(1-3 g \frac{\Phi^{2}}{\pi^{2}}\right)
$$

where $\varepsilon_{\infty}$ is the energy density in the thermodynamic limit. Thus $N \Delta E=\pi v g / 2$,

independent of $N$, for the metallic state. In an insulator, on the other hand, we find localized levels and the system cannot react to a twist in the boundary condition, i. e. $N \Delta E$ is expected to decrease with system size. Considering the above model, in which the contributions of the local potential and the weakening of the neighbouring bonds are taken into account separately, the localized levels do not split off the continuous spectrum [26], and the phase sensitivity is an appropriate observable. 


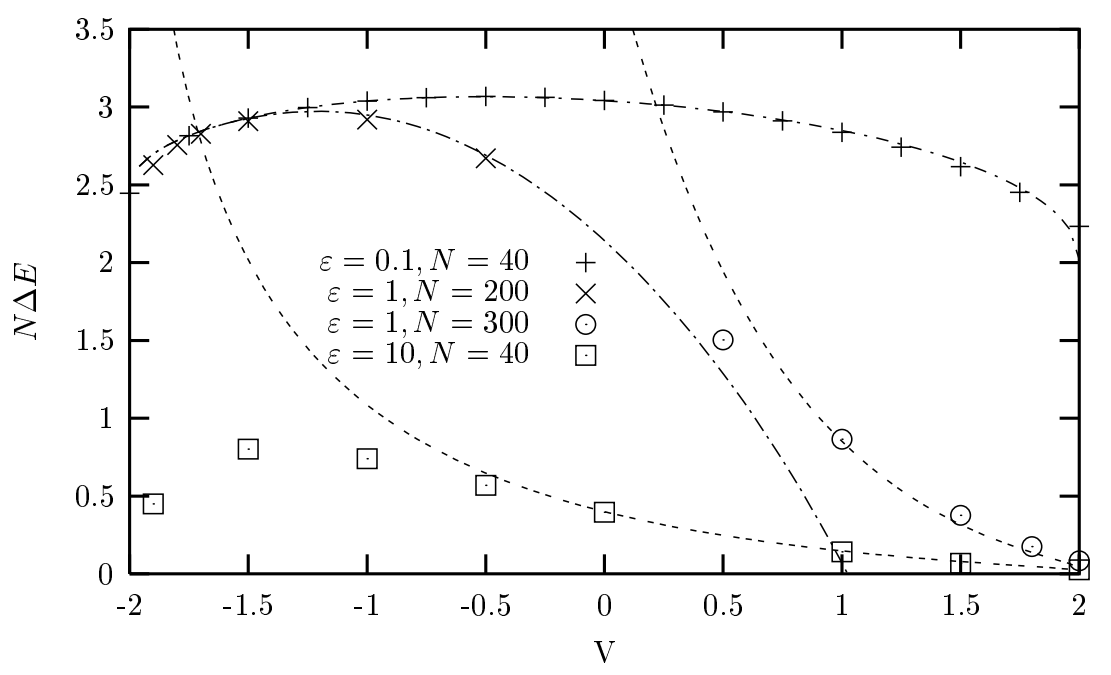

Fig. 1 Phase sensitivity as a function of interaction for a system with one site-impurity. The dashed-dotted lines correspond to Eq. 201, and the dashed lines to (21).

\section{Comparison of site- and bond-impurities}

\subsection{Local potential}

A local magnetic field, which corresponds to a local potential, is the well-known and most studied example of a site-impurity. In this case, the free motion of the fermions inside the ring is restricted by the back-scattering at the impurity $\left( \pm k_{F} \rightarrow \mp k_{F}\right)$. As discussed by Kane and Fisher [22] the impurity becomes transparent for an attractive, and completely reflective for a repulsive interaction, according to the renormalization group equation

$$
\frac{\mathrm{d} \epsilon_{0}}{\mathrm{~d} l}=(1-g) \epsilon_{0} .
$$

In other words, the impurity strength scales either to zero or to infinity, for $g>1$ and $g<1$, respectively. The fact that an impurity scales to zero for repulsive interaction is confirmed by the scaling of a weak link [22]. For strong $\epsilon_{0}$ the effective hopping is given by $t_{t}=4 t^{2} / \epsilon_{0}$. The final result for the phase sensitivity is obtained by perturbation theory with respect to the defect strength, see 21] and [27, and is given by

$$
N \Delta E=\frac{\pi v g}{2}-\epsilon_{0}\left(\frac{N}{N_{0}}\right)^{1-g}
$$

in the weak scattering limit, and by

$$
N \Delta E=\frac{4 t^{2}}{\left|\epsilon_{0}\right|}\left(\frac{N}{N_{0}}\right)^{1-1 / g}
$$


in the strong impurity limit, where $N_{0} \approx 2$ is a cut-off corresponding to a momentum cut-off of the order of the Fermi momentum. As a summary, we show numerical data in Fig. 1. The most important result is that an intermediate defect strength scales to zero for attractive interaction, and to infinity for repulsive interaction, rather than scaling to an additional intermediate fixed point.

\subsection{One modified link}

We begin by checking whether the above expressions for the weak and strong potential, Eqs. (20) and (21), can be applied to the case of a modified link, as should be expected from the treatment in [22] or 23]. We therefore calculate the phase sensitivity for a weakly modified link, $\delta_{t}=0.2$, and a weak link, $\delta_{t}=0.8$. The results are shown in Fig. 2. However, the above expressions do not apply: Instead of Eq. (20) we find for the weakly modified link

$$
N \Delta E=\frac{\pi v g}{2}-2 \delta_{t}\left(\frac{N}{N_{0}}\right)^{(1-g) / 2} .
$$

This is actually the scaling according to Eq. (14). Therefore, Eq. (12) does not appropriately describe this impurity type. Similarly, for the weak link, we do not recover Eq. (21), but instead

$$
N \Delta E=4\left(1-\delta_{t}\right)\left(\frac{N}{2 N_{0}}\right)^{(1-1 / g) / 2} .
$$

In this case $N \rightarrow N / 2$, as we connect two chains of length $N / 2$.

Next we verify that the generic link-impurity can be written as a sum of a hoppingand an interaction-impurity, as the bosonized Hamiltonians, Eqs. (14) and (16), suggest. As shown on the 1. h. s. of Fig. 3, where the three cases: $\delta_{t}=\delta$ and $\delta_{V}=0$, $\delta_{t}=\delta_{V}$, and $\delta_{V}=\delta$ and $\delta_{t}=0$, are compared, this conjecture is indeed confirmed. The qualitative behaviour is the same for all defect types. By comparing two different system sizes, we can fix the transition point to $V=0$ in all cases.

If the impurity is so strong that the sign of the hopping or the interaction changes, $\delta>1$, interaction dependent maxima occur, in addition, for the generic link-impurity, see the r. h. s. of Fig. 3 .

\subsection{Bond-impurity}

For the non-interacting system $(V=0)$ with one bond-impurity, it is easily seen that the back-scattering contributions, i. e. the Fourier components with $q=\pi$ in the halffilled case, cancel each other as is the case for two site-impurities at an odd distance. For this reason, we presume that the phase sensitivity increases slightly with system size according to $N \Delta E \propto\left(1-2 b N_{0} / N\right)$ also in the interacting system. For repulsive interaction the results of Eggert \& Affleck [3] show that this kind of perturbation is irrelevant and the system remains metallic. For an attractive interaction, the behaviour is expected to be similar. Accordingly, the results for integrable, triangle like defects [6] show that the phase sensitivity is slightly reduced for non-interacting fermions in 


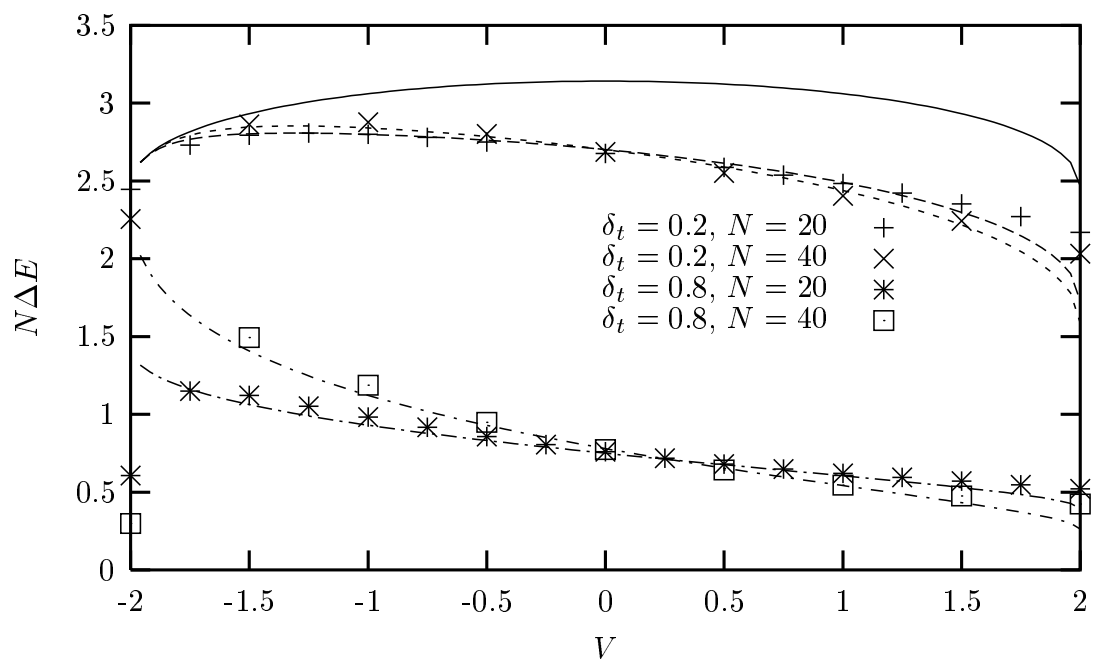

Fig. 2 Phase sensitivity as a function of interaction for a system with a hopping impurity. The straight line corresponds to the clean system, Eq. (18). The dashed lines correspond to Eq. (22), and the dashed-dotted lines to Eq. (23).

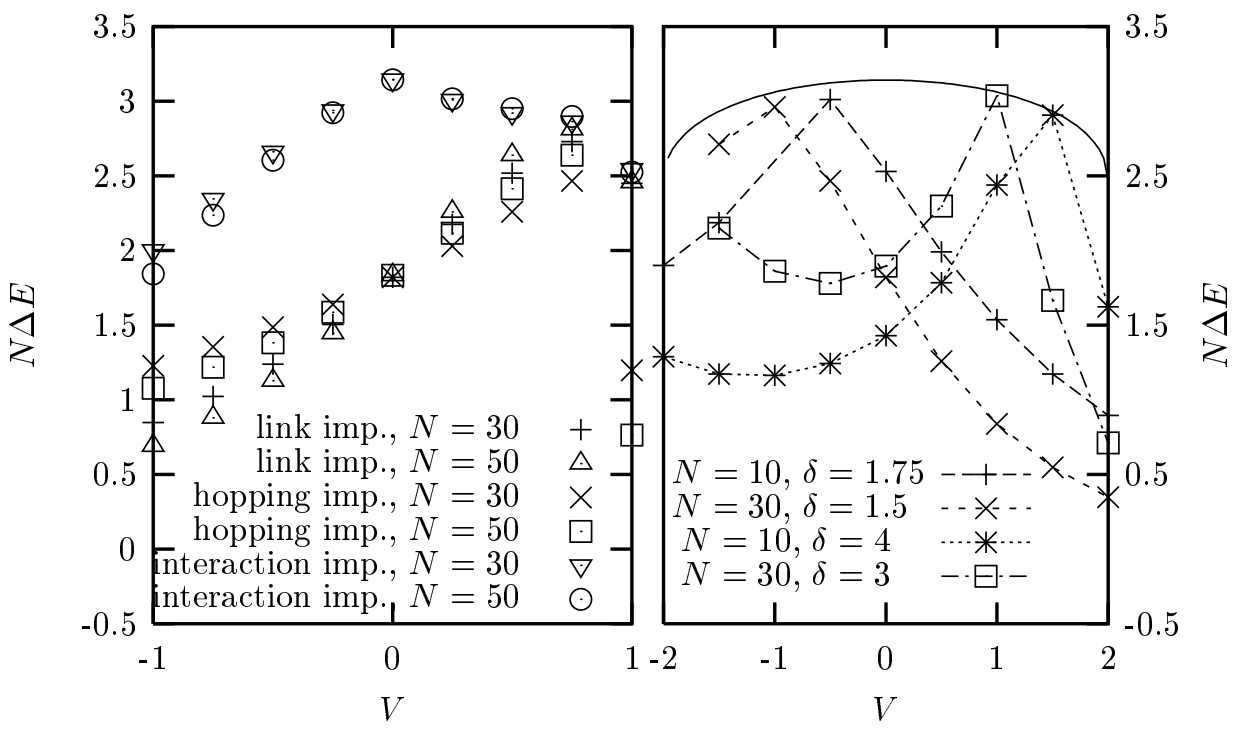

Fig. 3 Left: Phase sensitivity as a function of interaction for a system with one site-impurity of type $t, t V$, and $V$. Right: Details of the phase sensitivity as a function of interaction for a system with one strong link-impurity. The straight line corresponds to the clean case, the other lines are connecting the data points. 

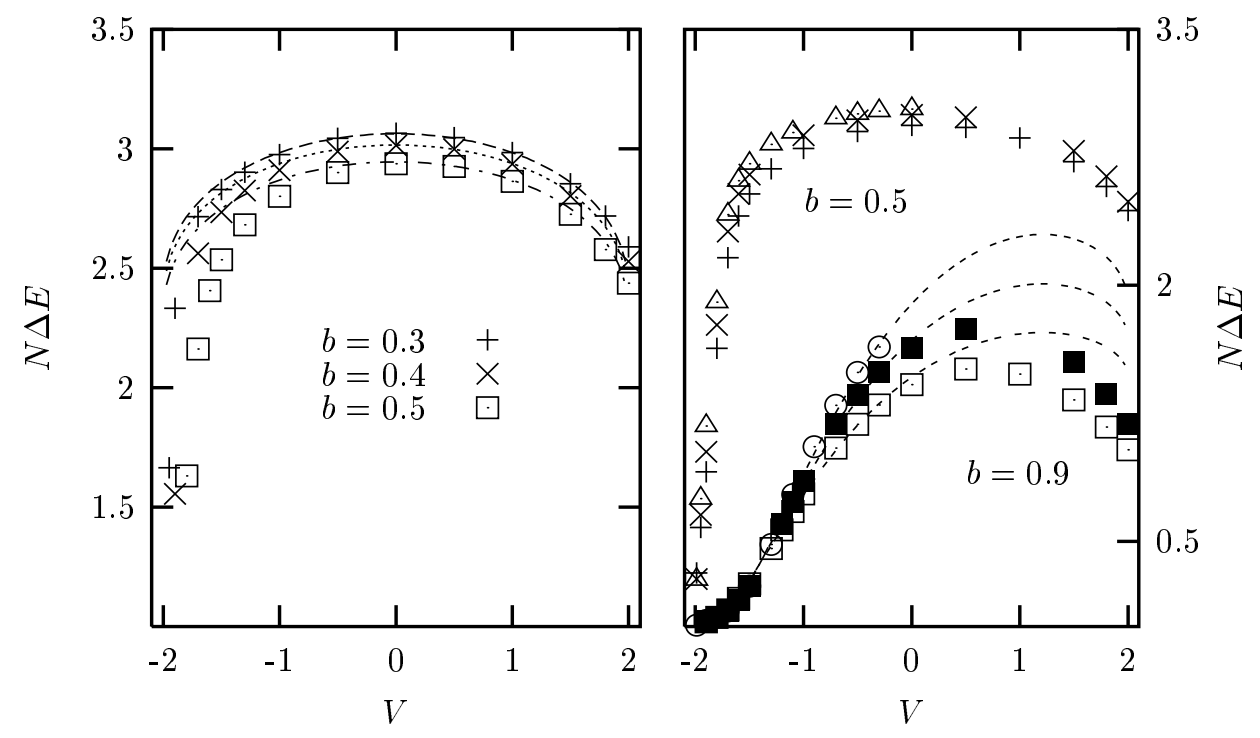

Fig. 4 Left: Phase sensitivity as a function of interaction for a system with one bond impurity of varying strength, the system size is $N=40$. The lines correspond to $N \Delta E(b=$ $0)\left(1-2 b l_{0} / N\right)$. Right: Phase sensitivity as a function of interaction for a system with one bond impurity of strength $b=0.5(N=40:+, N=60: \times, N=80: \triangle)$, and $b=0.9$ $(N=40: \square, N=60: \square, N=80$ : $\square$ ). The lines correspond to fits according to Eq. (24).

the presence of such (transparent) impurities. In particular, a repulsive interaction leads to stronger suppression of the phase sensitivity than an attractive one.

The numerical data presented in the left part of Fig. A confirm this assertion of an only slight reduction: clearly the system behaves metallic for small $b$. However, by increasing the impurity strength, strong deviations are visible, already for $b=0.3$ at $V=-1.9$. The value of the interaction, where this strong decrease is seen, moves to a larger interaction when increasing the impurity strength, until the maximum reaches the repulsive regime for $b>0.7$. Using the scaling applicable for the weak link case, Eq. (21), $t_{t} \rightarrow t_{t}^{\text {eff }}=t_{t} N^{1-1 / g}$, a scaling relation for the two-weak-link case, given by the Hamiltonian in Eq. (17), can be derived,

$$
(1-b) \rightarrow(1-b)_{\mathrm{eff}} \propto(1-b) N^{1-g / 2-1 /(4 g)} .
$$

According to this scaling relation, the weak links increase for $V>-1.2$. Thus, for an attractive interaction, there is an interaction value for which the phase sensitivity becomes independent of system size, while it scales to zero when further lowering the interaction down towards $V=-2$ (where the first-order transition takes place in the clean system). Nevertheless, the system remains metallic in this region, but with a strongly reduced Drude weight. This behaviour, see the r. h. s. of Fig. 烏 is different from the behaviour of an integrable defect (where a strong reduction arises for a repulsive interaction). 


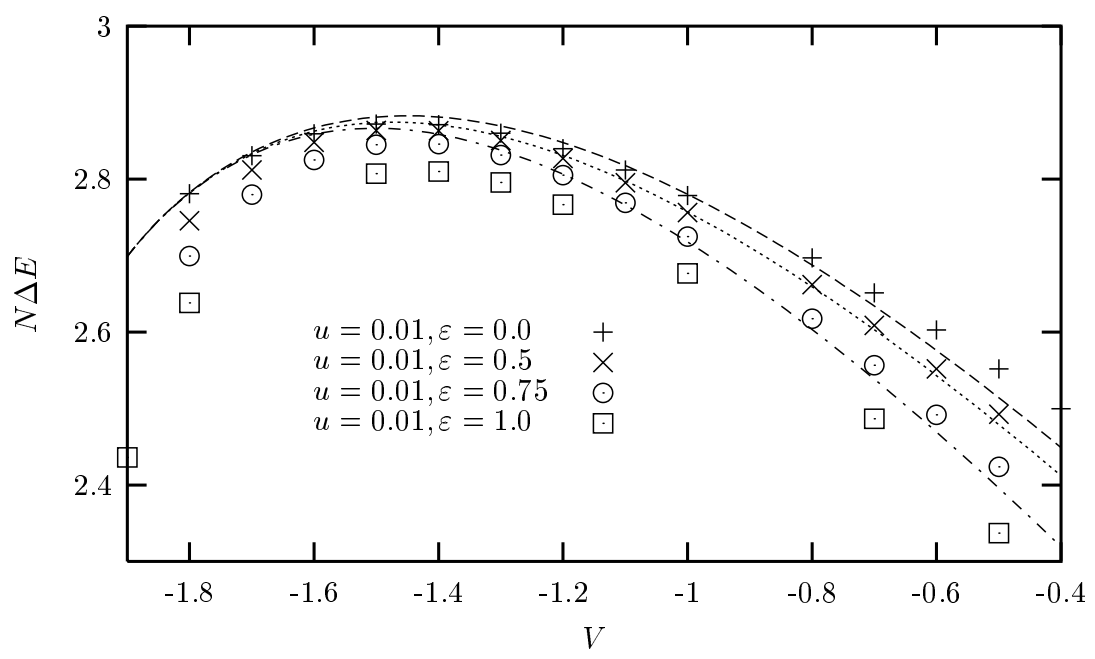

Fig. 5 Phase sensitivity versus interaction for a clean and a distorted dimerized system; the system size is $N=48$. The lines correspond to Eq. (26).

\section{Combination of dimerization and impurity}

\subsection{A barrier in a dimerized system}

We combine now two perturbations and study first a site-impurity in the dimerized system. For a first impression, we calculate the energy levels and the ground-state energy of of non-interacting particles with an alternating hopping, following the treatment in [28], but adding one potential scatterer of strength $\epsilon$. The ground-state energy is given by

$$
E=-2 t \sum_{-k_{F}}^{k_{F}} \sqrt{\cos ^{2} k a+\frac{\epsilon^{2}}{4 N^{2}}+u^{2} \sin ^{2} k a} \propto \mathrm{E}(\kappa)
$$

with $\mathrm{E}(\kappa)$ the second complete elliptic integral, where $\kappa$ is given by $\kappa^{2}=\left(1-u^{2}\right) /(1+$ $\left.\epsilon^{2}(N)\right) \sim 1-u^{2}-\epsilon^{2}(N) ; \epsilon(N)=\epsilon / 2 N$. A single impurity in the non-interacting alternating chain was also discussed in [29]. Thus the phase sensitivity is given by, compare [20]:

$$
N \Delta E=N \Delta E(0,0)-2 N_{0} \sqrt{u^{2}+\epsilon^{2}(N)}\left(\frac{N}{N_{0}}\right)^{2-g} .
$$

Figure 5 shows numerical data in comparison to this formula, with $N_{0} \approx 4$ according to the cell doubling in the dimerized state.

The length dependence presently is not as clear as for a single impurity or for the clean dimerized system: For $g<1$ the system is completely localized, the phase sensitivity decreases with system size, while for $g>2$, the system appears to be delocalized. In between, for $1<g<2$, however, a characteristic system size $N_{c}^{2}=$ 


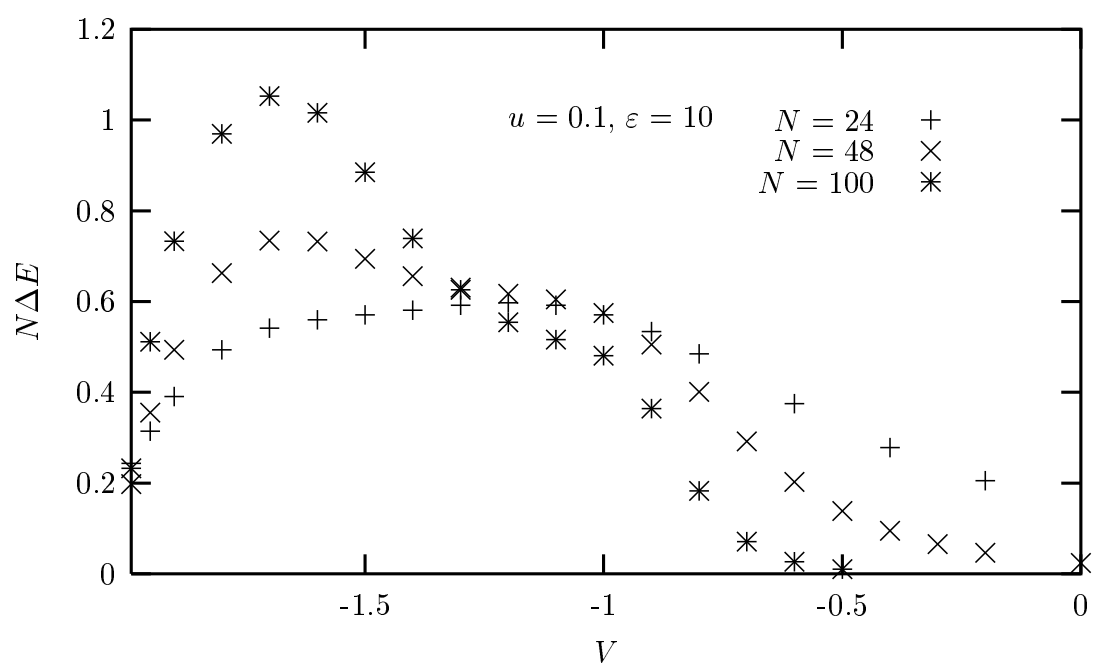

Fig. 6 Phase sensitivity as a function of interaction for a system with $u=0.1$ and $\epsilon=10$. For $V>-1$ the phase sensitivity decreases with system size, and for $V<-1.4$ it increases. In between, it increases from $N=24$ to $N=48$, but decreases from $N=48$ to $N=100$.

$\epsilon^{2}(g-1) /\left[4 u^{2}(2-g)\right]$ can be defined. For $N$ smaller than $N_{c}$, the phase sensitivity increases with $N$ similar to the behaviour in the delocalized phase. Increasing the system size above $N_{c}$, however, the phase sensitivity decreases, indicative of localization. This intermediate regime is shown in Fig. 6, where we plot the phase sensitivity for a set of parameters which clearly shows the described behaviour.

An impurity with weak or intermediate strength enlarges the delocalized region of the dimerized model, as already shown in [30]. For a strong barrier and a strong dimerization, a new feature is observed: In addition to the enlargement of the delocalized region a peak in the phase sensitivity is found in the localized regime, for $\epsilon>3$ and $u>0.1$. The characteristic behaviour is shown in Fig. 18, demonstrating that the increase for a value of $V_{\text {peak }} \approx-1$ is already by one order of magnitude for $N=100$. The assumption that the impurity becomes irrelevant at $V_{\text {peak }}$ is consistent

\begin{tabular}{|c|c||c|c|c|c||c|c|}
\hline$u_{V}=0$ & & & & $u_{V}=u_{t}$ & & & \\
$u$ & $\epsilon$ & $V_{\text {peak }}$ & $V_{c}$ & $u$ & $\epsilon$ & $V_{\text {peak }}$ & $V_{c}$ \\
0.1 & 10 & $(-1)$ & -1.3 & 0.1 & 10 & & -0.9 \\
0.15 & 10 & -1.1 & -1.4 & 0.2 & 10 & & -1.3 \\
0.2 & 10 & -1.2 & -1.6 \\
0.25 & 10 & -1.2 & -1.7 & 0.3 & 10 & -1.0 & -1.45 \\
0.3 & 10 & -1.2 & -1.8 & 10 & -1.05 & -1.65 \\
0.7 & 10 & -1.1 & -1.75 \\
\hline
\end{tabular}

Table 1 Values of $V_{\text {peak }}$ and $V_{c}$ for $u_{V}=0$, and $u_{V}=u_{t}$. The latter case is included for completeness, compare the discussion in subsection 2.2 , even though not mentioned separately in the text. 


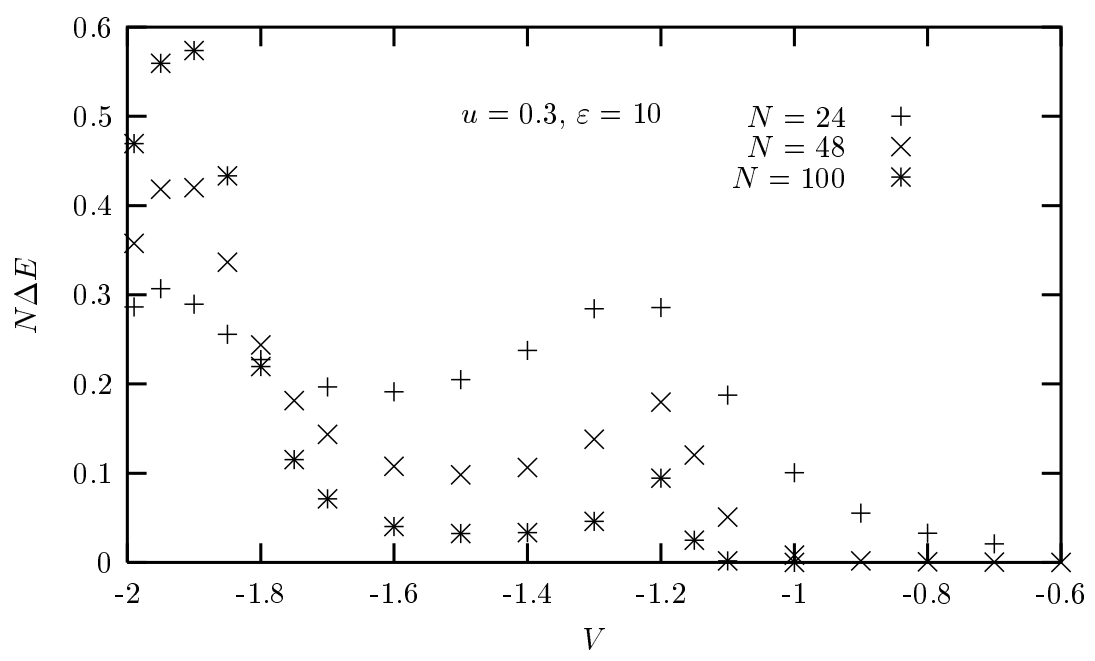

Fig. 7 Typical plot of the phase sensitivity versus interaction for a system with strong dimerization and a strong impurity.

with the initial increase of the phase sensitivity at this value. Since the effect of the dimerization is weakened by an impurity, the following (by further lowering the interaction down to the value $V_{c}$ ) decrease of the phase sensitivity can be explained by the enhancement of the effective dimerization, caused by the diminished influence of the impurity. Lowering the interaction further, the dimerization becomes irrelevant, too, and the system delocalizes. Table 1 shows the characteristic interaction values for this model.

\subsection{Dimerization and bond-impurity}

While we analyzed above a single site-impurity in a dimerized ring, we now concentrate on a single bond-impurity plus dimerization. Based on the results of Sec. 3.3, we expect that for a weak distortion the system still shows the phase transition at $V=$ $-\sqrt{2}$, with the delocalized region extending to $V=-2$. Increasing the strength of the bond-impurity, we also expect the unusual behaviour - as described above for strong attractive interaction to occur in the dimerized system as well, because in this interaction regime the dimerization is irrelevant. The first conjecture can be confirmed numerically for strong dimerization but small $b$. The numerical data for stronger distortion, i. e. for increasing $b$, show a more complex behaviour of the phase sensitivity, see Fig. 8. The first observation is, similar to a site-impurity, that the transition to the metallic state occurs at weaker interaction strength, e. g. for $u=0.03$ already at $V=-1$ (compared to $V \approx-1.5$ in the undistorted case). Also, the second conjecture is supported by the numerical data: For an interaction larger than $V \approx-1.5$ the influence of the bond-impurity drives the systems again to a metallic state with a strongly reduced phase sensitivity. In between, $-1.5<V<-1$, the system flows to the free metallic fixed point when increasing the system size. 


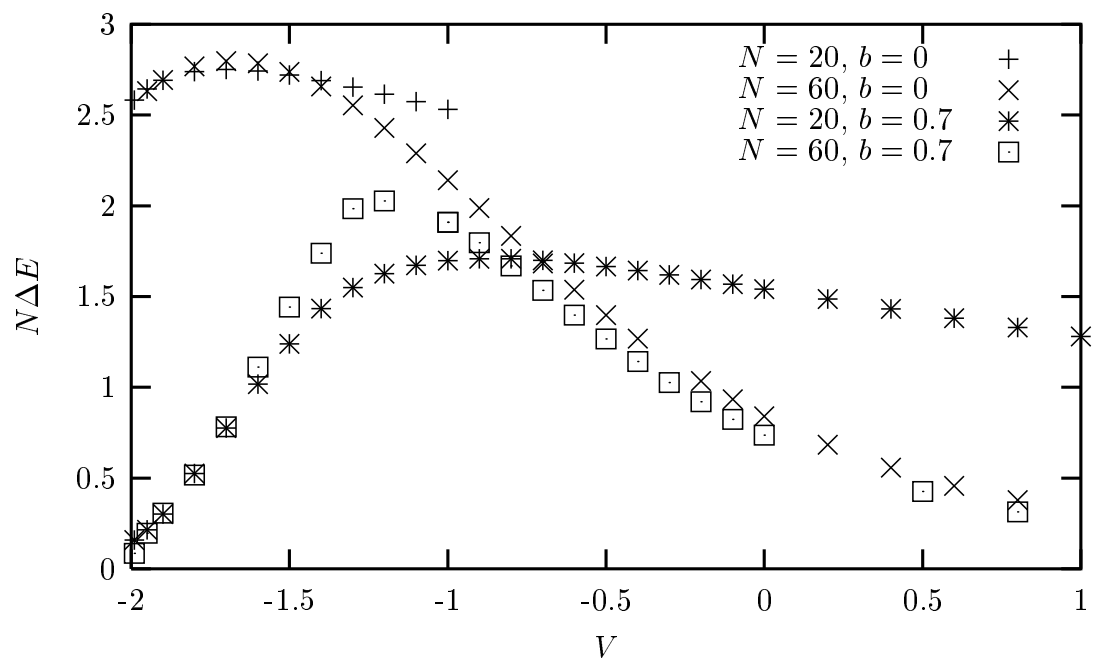

Fig. 8 Phase sensitivity as a function of interaction for a dimerized $(u=0.03)$ system with a strong bond-impurity, $b=0.7$; the system sizes are $N=20$ and $N=60$, respectively. For comparison, the data of the clean dimerized system, again for $N=20$ and 60 , are included.

\section{Summary}

Our numerical studies of a one-dimensional spin chain, which is equivalent to a system of spinless interacting fermions, with various distortions show that both types of siteimpurities are irrelevant for an attractive interaction (using the fermion picture), and relevant for a repulsive one. The description within a first-order perturbation treatment can be applied in the case of the site-impurity. The bond-impurity is irrelevant for repulsive interaction, and leads to a strong suppression of quantum coherence - but not to a localized ground-state for attractive interaction near the first-order transition. The scaling could be determined using the bosonization technique. In the dimerized system, the numerical results show that dimerization and any kind of impurity weaken each other. A single barrier in a dimerized chain leads to an enhancement of the delocalized region compared to the clean dimerized system. In addition, a sharp peak (as a function of the interaction parameter), increasing the phase sensitivity by one to two orders of magnitude for small systems, $N \approx 100$, is found for strong perturbations.

A bond-impurity in the dimerized system is irrelevant for a small impurity strength. A stronger impurity leads to a complicated phase diagram, especially for an attractive interaction. The combination of dimerization and impurity therefore leads in all cases to a shift of the localization-delocalization transition of the dimerized system to a weaker interaction strength. By tuning the values of dimerization and impurity strength, different metallic windows in the insulating system can be opened. At an interaction of about $V \approx-0.5, \ldots,-1$ a bond-impurity turns the system into the metal. For a strong attractive interaction, $V \approx-1.5, \ldots,-2$, where the bond-impurity reduces the Drude weight drastically, a strong site-impurity leads to delocalization, es- 
pecially in the strongly dimerized system.

The authors gratefully acknowledge financial support by the Deutsche Forschungsgemeinschaft (through SFB 484 and SPP 1073).

\section{References}

[1] J. G. Lussier et al., J. Phys. Cond. Matt. 7 (1995) L325; J. P. Renault et al., Europhys. Lett. 30 (1995) 475; N. C. Martin et al., Phys. Rev. B 56 (1997) 3173

[2] Y. J. Wang, V. Kiryukhin, R. J. Birgeneau, T. Masuda, I. Tsukada, and K. Uchinokura, Phys. Rev. Lett. 83 (1999) 1676; Y. J. Wang, Y.-J. Kim, R. J. Christianson, S. C. LaMarra, F. C. Chou, and R. J. Birgeneau, cond-mat/0012004

[3] S. Eggert and I. Affleck, Phys. Rev. B 46 (1992) 10866

[4] S. Qin, M. Fabrizio, L. Yu, M. Oshikawa, and I. Affleck, Phys. Rev. B 56 (1997) 9766

[5] V. Meden, P. Schmitteckert, and N. Shannon, Phys. Rev. B 57 (1998) 8878

6] P. Schmitteckert, P. Schwab, and U. Eckern, Europhys. Lett. 30 (1995) 543

[7] A. Kl" umper and A. A. Zvyagin, Phys. Rev. Lett. 81 (1998) 4975; A. A. Zvyagin, Phys. Rev. B 62 (2000) R6069

[8] J. Igarashi, T. Tonegawa, M. Kaburagi, and P. Fulde Phys. Rev. B 51 (1995) 5814; W. Zhang, J. Igarashi, and P. Fulde Phys. Rev. B 56 (1997) 654

[9] R. A. Hyman, Kun Yang, R. N. Bhatt, and S. M. Girvin, Phys. Rev. Lett. 76 (1996) 839

[10] N. Fabrizio and R. M'elin, Phys. Rev. Lett. 78 (1997) 3382

[11] N. Mostovoy and D. Khomskii, Z. Phys. B 103 (1997) 209; M. Mostovoy, D. Khomskii, and J. Knoester, Phys. Rev. B 58 (1998) 8190

[12] L. Balents and M. P. A. Fisher, Phys. Rev. B 56 (1997) 12970

[13] M. Steiner, M. Fabrizio, and A. O. Gogolin, Phys. Rev. B 57 (1998) 8290; A. O. Gogolin, A. A. Nersesyan, A. N. Tsvelik, and L. Yu, Nucl. Phys. B 540 (1999) 705

[14] U. Eckern and K. Ziegler, J.Phys.: Condens. Matter 10 (1998) 6749

[15] S. R. White, Phys. Rev. Lett. 69 (1992) 2863; S. R. White and R. N. Noack, Phys. Rev. Lett. 68 (1992) 3487; S. R. White, Phys. Rev. B 48 (1993) 10345

[16] F. D. M. Haldane, Phys. Rev. Lett. 47 (1981) 1840

[17] R. E. Peierls, Quantum Theory of Solids, Oxford University Press, Oxford, 1955

[18] M. Kohmoto, M. den Nijs, and L. P. Kadanoff, Phys. Rev. B 24 (1981) 5229

[19] F. C. Alcaraz and A. L. Malvezzi, J. Phys. A: Math. Gen. 28 (1995) 1521

20] C. Schuster and U. Eckern, Eur. Phys. J. B 5 (1998) 395

[21] C. Schuster, PhD thesis, Shaker Verlag, Aachen, 1999

[22] C. L. Kane and M. P. A. Fisher, Phys. Rev. Lett. 68 (1992) 1220

[23] C. A. Doty and D. S. Fisher, Phys. Rev. B 45 (1992) 2167

[24] F. Woynarovich and H. P. Eckle, J. Phys. A 20 (1987) L97

[25] B. S. Shastry and B. Sutherland, Phys. Rev. Lett. 65 (1990) 243 B. Sutherland and B. S. Shastry, Phys. Rev. Lett. 65 (1990) 1833

[26] V. Z. Kleiner and V. M. Tsukernik, Sov. J. Low Temp. Phys. 6 (1980) 158

[27] P. Schmitteckert, T. Schulze, C. Schuster, P. Schwab, and U. Eckern, Phys. Rev. Lett. 80 (1998) 560

[28] W. P. Su, J. P. Schrieffer, and A. J. Heeger, Phys. Rev. B 22 (1980) 2099

[29] A. A. Zvyagin and Y. Y. Segal, Low Temp. Phys. 21 (1995) 822

[30] C. Schuster and U. Eckern, Ann. Phys. (Leipzig) 8 (1999) 585 